\title{
Mutational Profiling of Brazilian Mucinous Adenocarcinoma of the Ovary
}

Lorena Ferrante ${ }^{1}$; Nathalia C Campanella2; Rozany M Dufloth; Carlos E M Andrade3;

Georgia C Fontes; Marcelo A Vieira ${ }^{3}$; Rui M V Reis²; Ricardo dos Reis ${ }^{*}$.

${ }^{1}$ Faculdade de Ciências da Saúde de Barretos Dr. Paulo Prata - FACISB, Barretos, São Paulo, Brazil;

${ }^{2}$ Molecular Oncology Research Center, Barretos Cancer Hospital, Barretos, São Paulo, Brazil;

${ }^{3}$ Gynecologic Oncology Department, Barretos Cancer Hospital, Barretos, São Paulo, Brazil.

\section{OBJECTIVE}

To investigate the mutational profiling associated with Brazilian mucinous adenocarcinoma of ovary.

\section{METHODS}

We included 47 patients from Barretos Cancer Hospital, from 2009 and 2015. HER2 amplification was evaluated by immunohistochemistry (IHC) and/or in situ hybridization (FISH). In addition, the hotspots regions of the KRAS (codons 12 and 13) were screened by PCR followed by direct Sanger sequencing. Finally, to provide a comprehensive assessment of 15 genes that are commonly mutated in solid tumors we performed the NGS-based mutation analysis using the Illumina TruSight Tumor 15 panel on MiSeq instrument (Illumina, USA).

\section{RESULTS}

HER2 IHC was performed in 28 samples and the negative staining was found in 26 (92.8\%). In 2 samples $(7.7 \%)$, because the IHC was inconclusive (moderate expression), we performed hybridization in situ (FISH) and found HER2 amplification in both. Clinically, both cases had pseudomixoma peritonei and 1 had disease recurrence. To KRAS Sanger sequencing, due to DNA quality issues, conclusive results were obtained in 26 cases. Interestingly, KRAS mutations were found in 11 samples (42.3\%), all in codon 12, and were associated with no recurrence $(p=0.036)$. By Illumina TruSight Tumor 15 panel we evaluated 16 samples. All samples showed at least one mutation in one gene and the most mutated genes were: TP53 and KRAS (10 mutations in each), BRAF (4) and HER2 and PIK3CA (1).

Table 1: Summary of results to HER2 IHC, KRAS Sanger sequencing and Illumina NGS TruSight Tumor 15.

\begin{tabular}{|c|c|c|c|}
\hline ID & HER2 IHC & $\begin{array}{l}\text { KRAS Sanger } \\
\text { Sequencing }\end{array}$ & IIlumina NGS \\
\hline 45 & neg $(0)$ & G12V & Not avaiable \\
\hline 16 & neg $(0)$ & G12A & Not avaiable \\
\hline 8 & neg (1) & WT & Not avaiable \\
\hline 25 & neg $(0)$ & Failed & Not avaiable \\
\hline 19 & neg $(0)$ & WT & KRAS - c. $183 A>C$ and TP53 - c.524G>A \\
\hline 20 & neg $(0)$ & WT & Not avaiable \\
\hline 12 & neg $(0)$ & WT & PIK3CA - c. $1634 A>G$ \\
\hline 40 & neg $(0)$ & Failed & Not avaiable \\
\hline 37 & neg $(0)$ & WT & TP53 - c.665C>T \\
\hline 5 & No core & Failed & Not avaiable \\
\hline 7 & neg $(0)$ & G12A & KRAS - c.35G>A and TP53 - c.559+1G>A \\
\hline 46 & neg $(0)$ & WT & Not avaiable \\
\hline 10 & neg $(0)$ & G12A & KRAS - c.35G>T and TP53 - c.374C>A \\
\hline 38 & neg $(0)$ & G12A & KRAS - c. $35 \mathrm{G}>\mathrm{T}$ and TP53 - c. $892 \mathrm{G}>\mathrm{T}$ \\
\hline 24 & neg $(0)$ & G12A & KRAS - c.35G $>T$ and TP53 $-\mathrm{c} .469 G>T$ \\
\hline 15 & doubt $(2+)$ & WT & TP53 - c.709_711delATG \\
\hline 43 & neg $(0)$ & G12A & KRAS- c.34G>C \\
\hline 27 & neg (1) & WT & BRAF - c.1799T>A and ERBB2 - c.2039C>T \\
\hline 29 & neg $(0)$ & G12A & KRAS - c.35G>T \\
\hline 31 & neg $(0)$ & WT & BRAF - c. $1801 A>G$ \\
\hline 30 & No tumor & G12A & Not avaiable \\
\hline 34 & No tumor & WT & Not avaiable \\
\hline 35 & neg (1) & G12C & Not avaiable \\
\hline 17 & neg $(0)$ & G12C & KRAS- c.34G>T and TP53 - c.817C>T \\
\hline 6 & neg $(0)$ & Failed & Not avaiable \\
\hline 26 & neg $(0)$ & WT & BRAF - c.1799T>A \\
\hline 41 & neg $(0)$ & Failed & Not avaiable \\
\hline 32 & doubt $(2+)$ & WT & BRAF - c. $1808 \mathrm{G}>\mathrm{A}, \mathrm{KRAS}-\mathrm{c} .35 \mathrm{G}>\mathrm{T}, \mathrm{KRAS}-\mathrm{c} .448 \mathrm{C}>\mathrm{T}$ and TP53 - c.796G>A \\
\hline 23 & No tumor & WT & TP53 - c.524G>A \\
\hline 2 & neg $(0)$ & Failed & Not avaiable \\
\hline 13 & neg $(0)$ & WT & Not avaiable \\
\hline 18 & neg $(0)$ & WT & Not avaiable \\
\hline
\end{tabular}

\section{CONCLUSIONS}

We identified the mutational profiling associated with Brazilian mucinous adenocarcinoma of ovary highlighting its genomic heterogeneity and potential therapeutic targets that could be investigated in future new clinical trials. 